Supplementary Materials for manuscript:

\title{
Anthocyanin profiling of maize grains using DIESI-MSQD reveals that cyanidin- based derivatives predominate in purple corn, whereas pelargonidin-based molecules occur in red-pink varieties from Mexico
}

Peniche-Pavía \& Tiessen 2020

- Sample information (Table S1-2)

- A visual explanation for the mass methods used (S1),

- Fragmentation patterns for anthocyanin ions (S2-10),

- Extra heatmaps (S11-S12)

- Boxplots for selected anthocyanins (S13-18).

\begin{tabular}{ccccccc}
\hline Code & mean \pm SD & Code & mean \pm SD & Code & mean \pm SD \\
\hline EO1_Pur & $0.59 \pm 0.03$ & EO3_Red & $0.42 \pm 0.02$ & VM2_Lpu & $0.28 \pm 0.02$ \\
EO1_Red & $0.54 \pm 0.03$ & EO3_Lpu & $0.48 \pm 0.05$ & VM2_Yew & $0.32 \pm 0.03$ \\
EO1_Lpu & $0.59 \pm 0.03$ & EO4_Bro & $0.41 \pm 0.05$ & VM3_Pur & $0.19 \pm 0.01$ \\
EO2_Pur & $0.50 \pm 0.12$ & EO4_Pur & $0.59 \pm 0.01$ & VM3_Lpu & $0.20 \pm 0.02$ \\
EO2_Red & $0.53 \pm 0.15$ & EO4_Red & $0.59 \pm 0.05$ & VM4_Bla & $0.19 \pm 0.01$ \\
EO2_Lpu & $0.54 \pm 0.08$ & CP1_Pur & $0.53 \pm 0.02$ & VM4_Pur & $0.17 \pm 0.02$ \\
EO2_Pin & $0.58 \pm 0.08$ & CA1_Yew & $0.37 \pm 0.06$ & VM4_Bro & $0.17 \pm 0.02$ \\
EO2_Lpi & $0.46 \pm 0.01$ & VM1_Pur & $0.21 \pm 0.02$ & CN1_Bla & $0.51 \pm 0.02$ \\
EO3_Bro & $0.52 \pm 0.03$ & VM2_Bla & $0.31 \pm 0.03$ & 366_Bla & $0.40 \pm 0.04$ \\
\hline EO3_Pur & $0.53 \pm 0.04$ & VM2_Pur & $0.31 \pm 0.02$ & ATF_Bla & $0.40 \pm 0.00$ \\
\hline
\end{tabular}

Table S1. Average weights in $g$ of individual kernels. The names of the landraces and Vitamaize entries and the phenotypic color were abbreviated and joined to form a code for subsequent analysis. The first part of the code corresponds to genotype: Elote Occicental (EO), Criollo Amarillo (CA1), Vitamaize (VM), Conico Negro (CN1). The second part of the code corresponds to the kernel color with following abbrViations: purple (Pur), light purple (Lpu), pink (Pin), light pink (Lpi), brown (Bro), yellow (Yew), and black (Bla). 
Dry weights in $\mathrm{g}$ of individual kernels. Raw data:

\begin{tabular}{lccclccc}
\hline Code & $\begin{array}{l}\text { Sample } \\
\text { I }\end{array}$ & $\begin{array}{l}\text { Sample } \\
\text { II }\end{array}$ & $\begin{array}{l}\text { Sample } \\
\text { III }\end{array}$ & Code & $\begin{array}{l}\text { Sample } \\
\text { I }\end{array}$ & Sample II & $\begin{array}{l}\text { Sample } \\
\text { III }\end{array}$ \\
\hline EO1_Pur & 0.576 & 0.568 & 0.626 & CP1_Pur & 0.525 & 0.507 & 0.558 \\
EO1_Red & 0.543 & 0.506 & 0.567 & CA1_Yew & 0.420 & 0.392 & 0.297 \\
EO1_Lpu & 0.611 & 0.561 & 0.588 & VM1_Pur & 0.200 & 0.200 & 0.231 \\
EO2_Pur & 0.64 & 0.438 & 0.418 & VM2_Bla & 0.332 & 0.283 & 0.328 \\
EO2_Red & 0.384 & 0.54 & 0.674 & VM2_Pur & 0.332 & 0.296 & 0.302 \\
EO2_Lpu & 0.525 & 0.563 & 0.544 & VM2_Lpu & 0.301 & 0.273 & 0.26 \\
EO2_Pin & 0.63 & 0.622 & 0.482 & VM2_Yew & 0.34 & 0.33 & 0.284 \\
EO2_Lpi & 0.449 & 0.466 & 0.457 & VM3_Pur & 0.189 & 0.191 & 0.202 \\
EO3_Bro & 0.486 & 0.552 & 0.519 & VM3_Lpu & 0.172 & 0.211 & 0.204 \\
EO3_Pur & 0.565 & 0.492 & 0.526 & VM4_Bla & 0.182 & 0.182 & 0.195 \\
EO3_Red & 0.402 & 0.444 & 0.403 & VM4_Pur & 0.186 & 0.151 & 0.181 \\
EO3_Lpu & 0.461 & 0.445 & 0.53 & VM4_Bro & 0.181 & 0.141 & 0.179 \\
EO4_Bro & 0.368 & 0.471 & 0.392 & CN1_Bla & 0.522 & 0.486 & 0.528 \\
EO4_Pur & 0.418 & 0.407 & 0.391 & 366_Bla & 0.359 & 0.436 & 0.403 \\
EO4_Red & 0.271 & 0.346 & 0.37 & ATF_Bla & 0.403 & 0.406 & 0.397 \\
\hline
\end{tabular}

Table S2. List of anthocyanins detected with the Neutral Loss Method (MS2 data).

\begin{tabular}{|lcc}
\hline \multicolumn{1}{c}{ Molecule } & $\begin{array}{c}\text { Fragment loss } \\
\text { of }\end{array}$ & $\begin{array}{c}\text { Parent } \\
\text { ion } \mathbf{m} / \boldsymbol{z}\end{array}$ \\
\hline cyanidin-3- $O$-glucoside & $162 \mathrm{Da}$ & 449 \\
pelargonidn-3-O-glucoside & $162 \mathrm{Da}$ & 433 \\
peonidin-3-O-glucoside & $162 \mathrm{Da}$ & 463 \\
cyanidin-3-O-(6"-malonyl-glucoside) & $248 \mathrm{Da}$ & 535 \\
pelargonidn-3-O-(6"-malonyl-glucoside) & $248 \mathrm{Da}$ & 519 \\
peonidin-3-O-(6"-malonyl-glucoside) & $248 \mathrm{Da}$ & 549 \\
cyanidin-3- $O$-(6"-succinyl-glucoside) & $262 \mathrm{Da}$ & 549 \\
pelargonidn-3-O-(6"-succinyl-glucoside) & $262 \mathrm{Da}$ & 533 \\
peonidin-3- $O$-(6"-succinyl-glucoside) & $262 \mathrm{Da}$ & 563 \\
cyanidin-3- $O$-(dimalonyl-glucoside) & $334 \mathrm{Da}$ & 619 \\
pelargonidn-3-O-(dimalonyl-glucoside) & $334 \mathrm{Da}$ & 605 \\
peonidin-3-O-(dimalonyl-glucoside) & $334 \mathrm{Da}$ & 635 \\
cyanidin-3- $O$-(malonyl, succinyl-glucoside) & $348 \mathrm{Da}$ & 635 \\
pelargonidn-3-O-(malonyl, succinyl-glucoside) & $348 \mathrm{Da}$ & 619 \\
peonidin-3- $O$-(malonyl, succinyl-glucoside) & $348 \mathrm{Da}$ & 649 \\
cyanidin-3- $O$-(disuccinyl-glucoside) & $362 \mathrm{Da}$ & 649 \\
pelargonidn-3- $O$-(disuccinyl-glucoside) & $362 \mathrm{Da}$ & 633 \\
peonidin-3- $O$-(disuccinyl-glucoside) & $362 \mathrm{Da}$ & 663 \\
\hline
\end{tabular}




\section{MS/MS protocols}

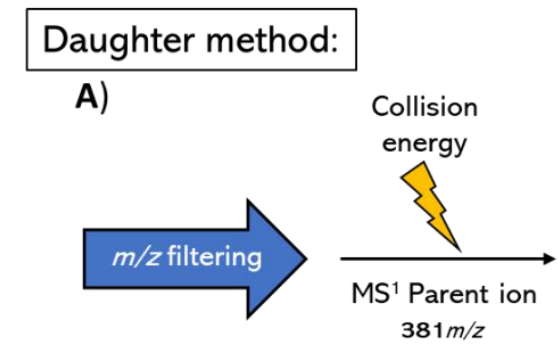

Neutral Loss method:

B)

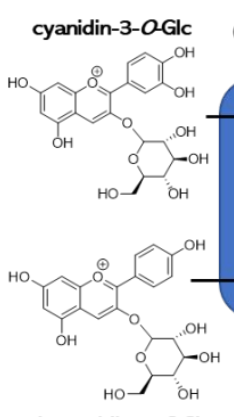

pelargonidin-3-OGlc

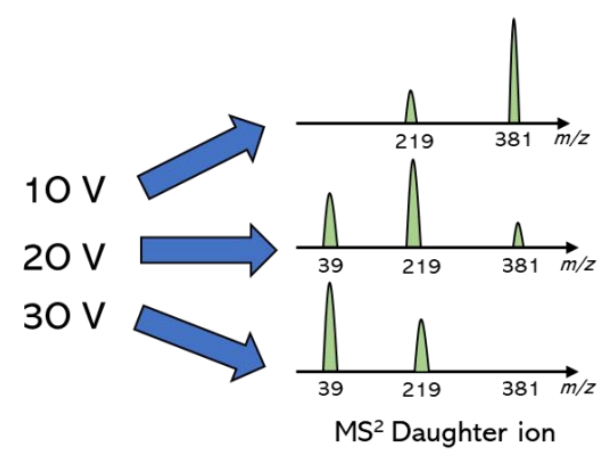

Collision energy

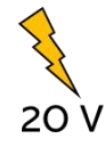

Fragmentation of glycosidic bond

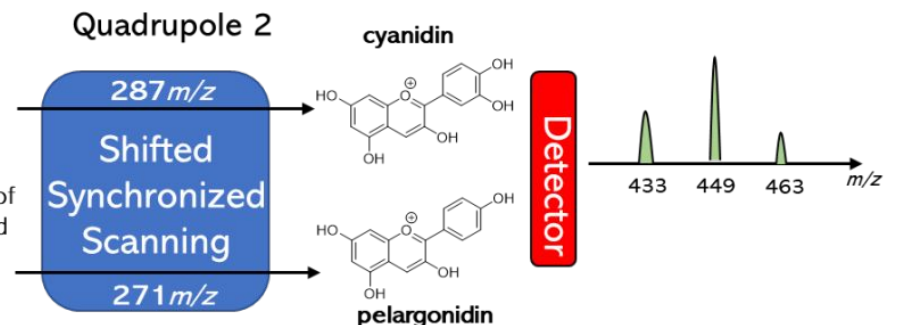

Figure S1. Overview of the two MS/MS methods employed to identify candidate ions. The combination of both methods allowed to tentatively assign ions a chemical structure and thus give them a name. 


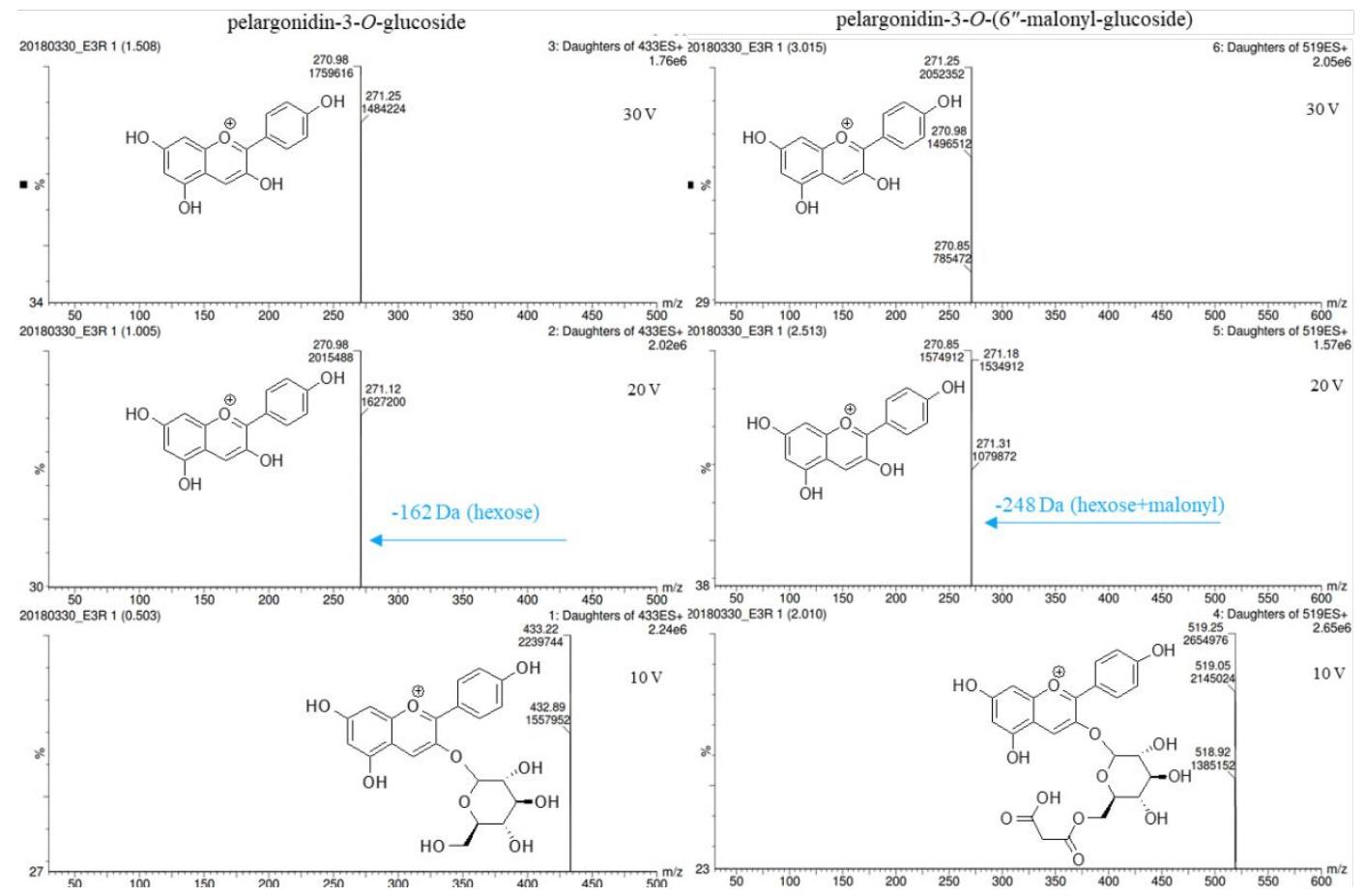

Figure S2. Mass spectra for pelargonidin-3-O-glucoside and pelargonidin-3-O-(6"-malonylglucoside). Data was obtained with Daughters method in MassLynx 4.1 using $10 \mathrm{~V}, 20 \mathrm{~V}$, and 30 V. Representative data is shown (sample EO3_Red).

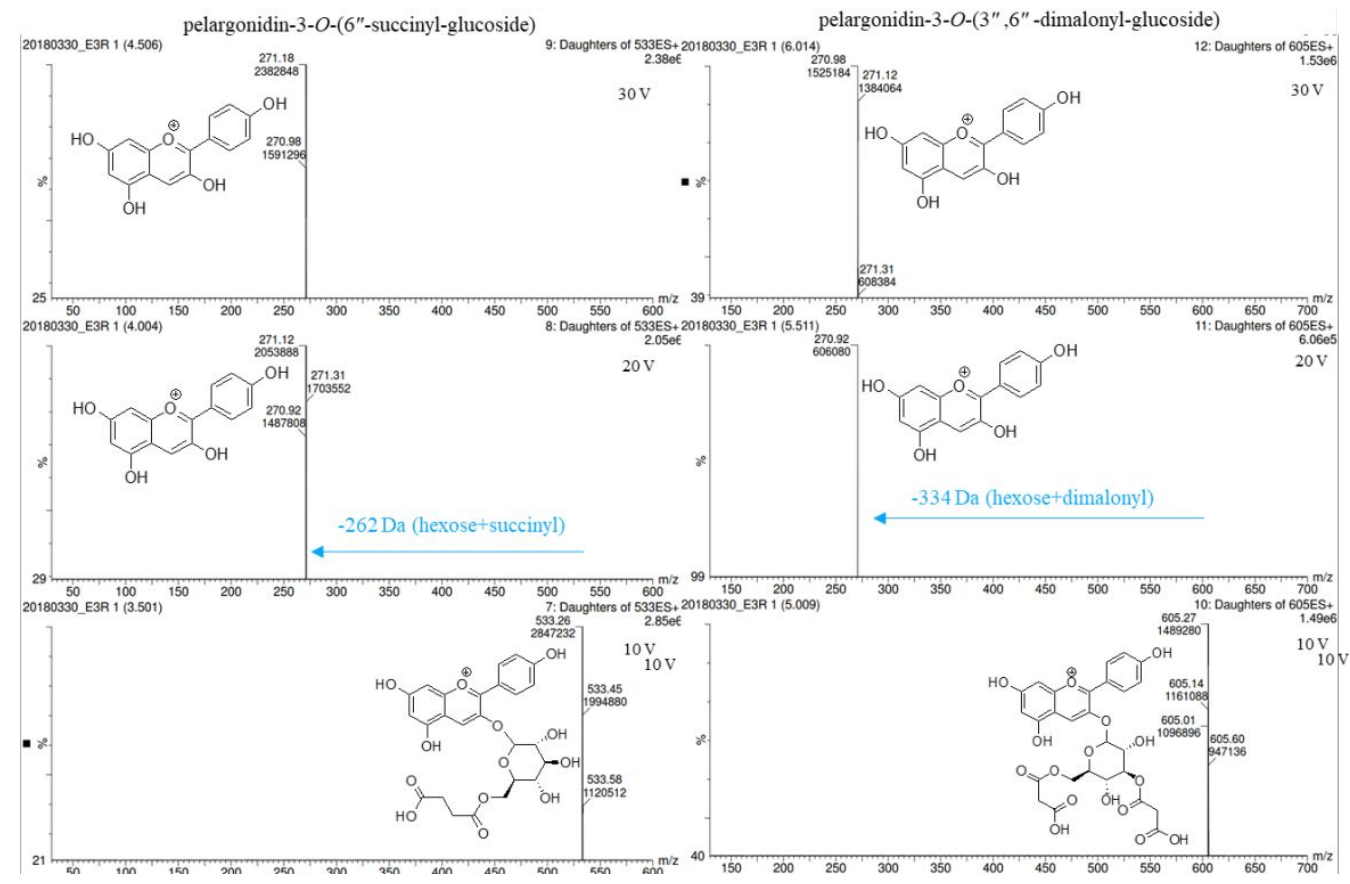

Figure S3. Mass spectra for pelargonidin-3-O-(6"-succinyl-glucoside) and pelargonidin-3-O(dimalonyl-glucoside). Data was obtained with Daughters method in MassLynx 4.1 using 10 V, 20 $\mathrm{V}$, and $30 \mathrm{~V}$. Representative data is shown (sample EO3_Red). 


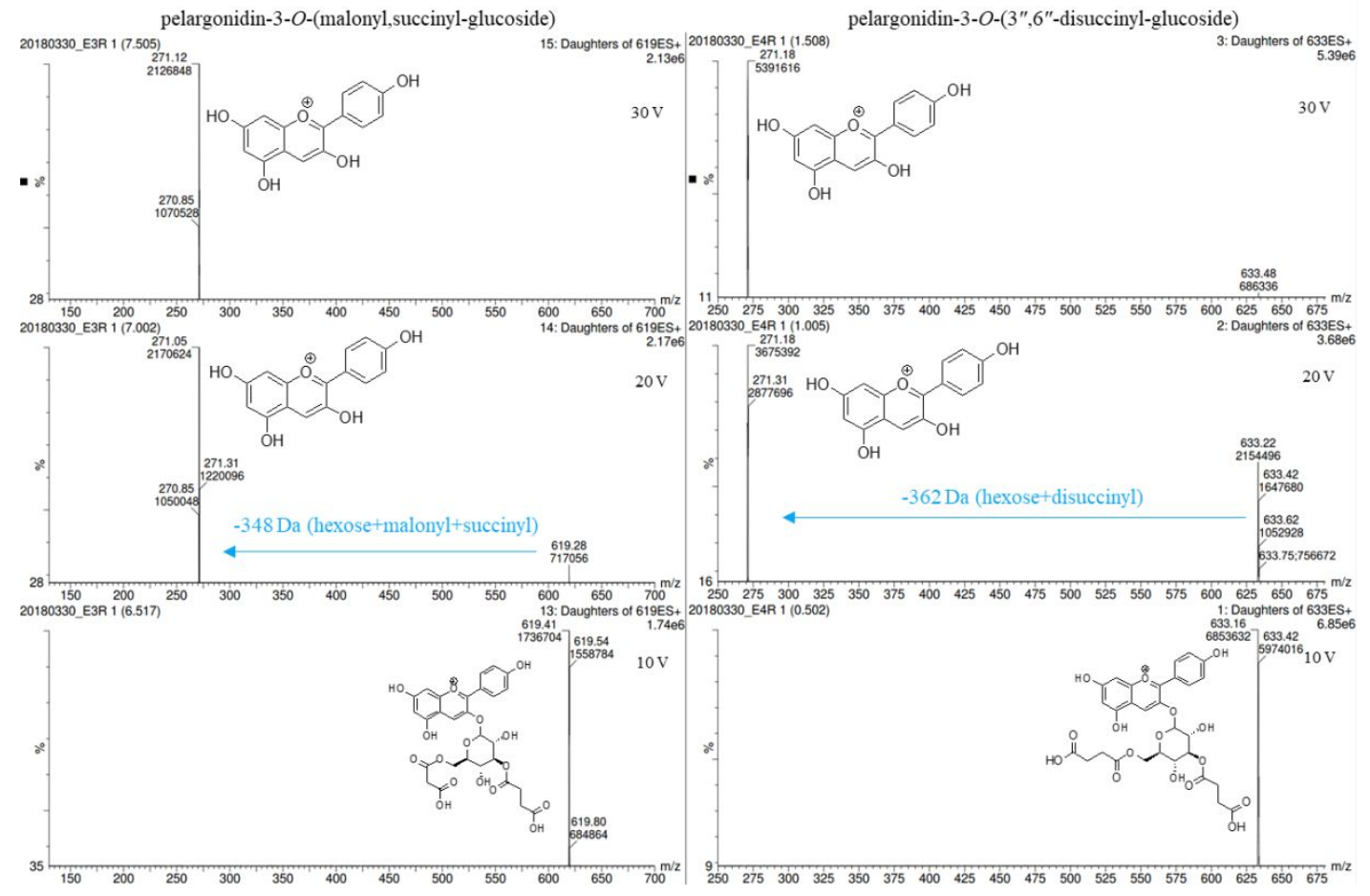

Figure S4. Mass spectra for pelargonidin-3- $O$-(malonyl, succinyl-glucoside) and pelargonidin-3-O(disuccinyl-glucoside). Data was obtained with Daughters method in MassLynx 4.1 using 10 V, 20 $\mathrm{V}$, and $30 \mathrm{~V}$. Representative data is shown (sample EO3_Red).

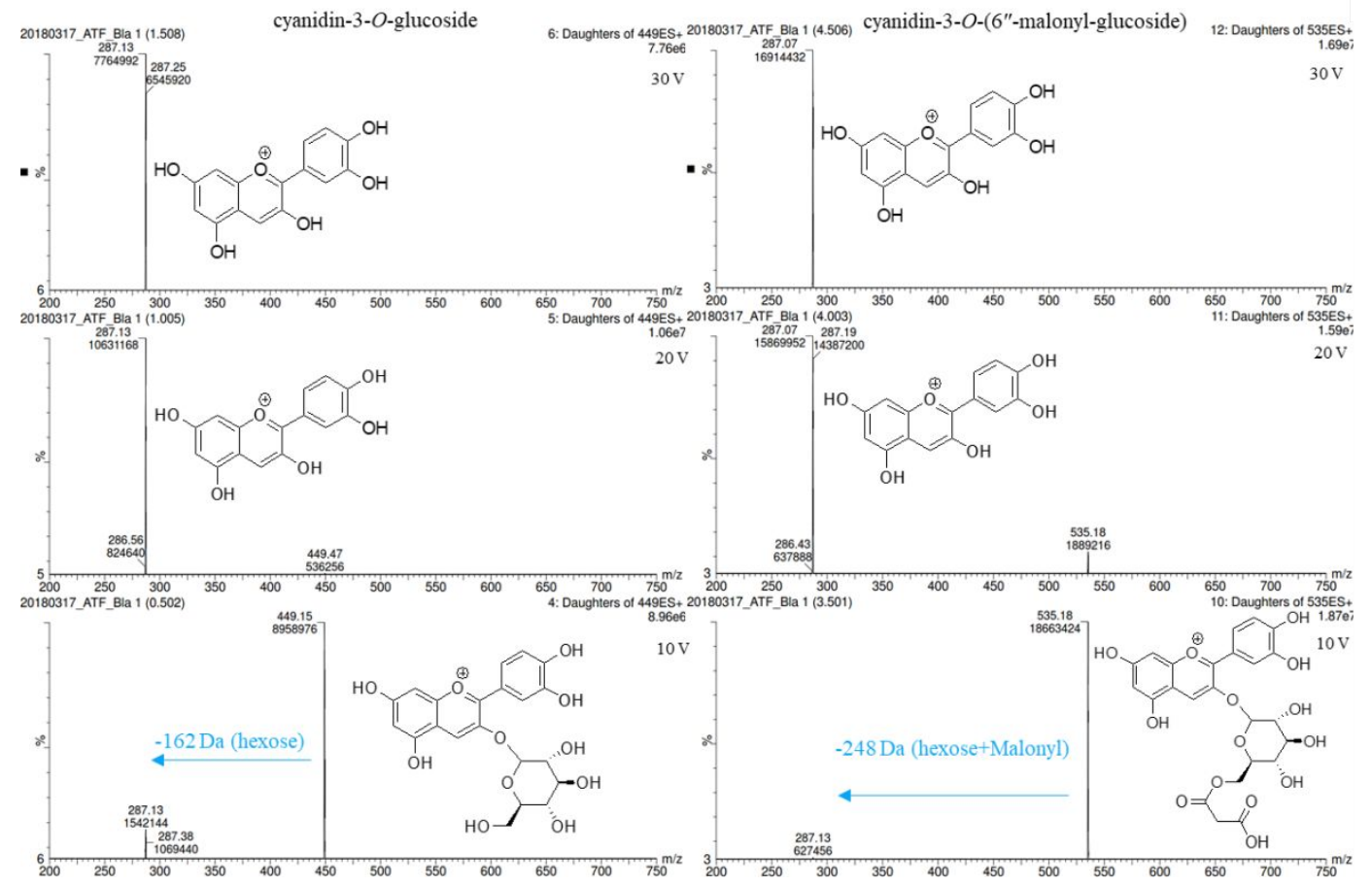

Figure S5. Mass spectra for cyanidin-3-O-glucoside and cyanidin-3- $O$-(6"-malonyl-glucoside). Data was obtained with Daughters method in MassLynx 4.1 using $10 \mathrm{~V}, 20 \mathrm{~V}$, and $30 \mathrm{~V}$. Representative data is shown (sample ATF_Black). 


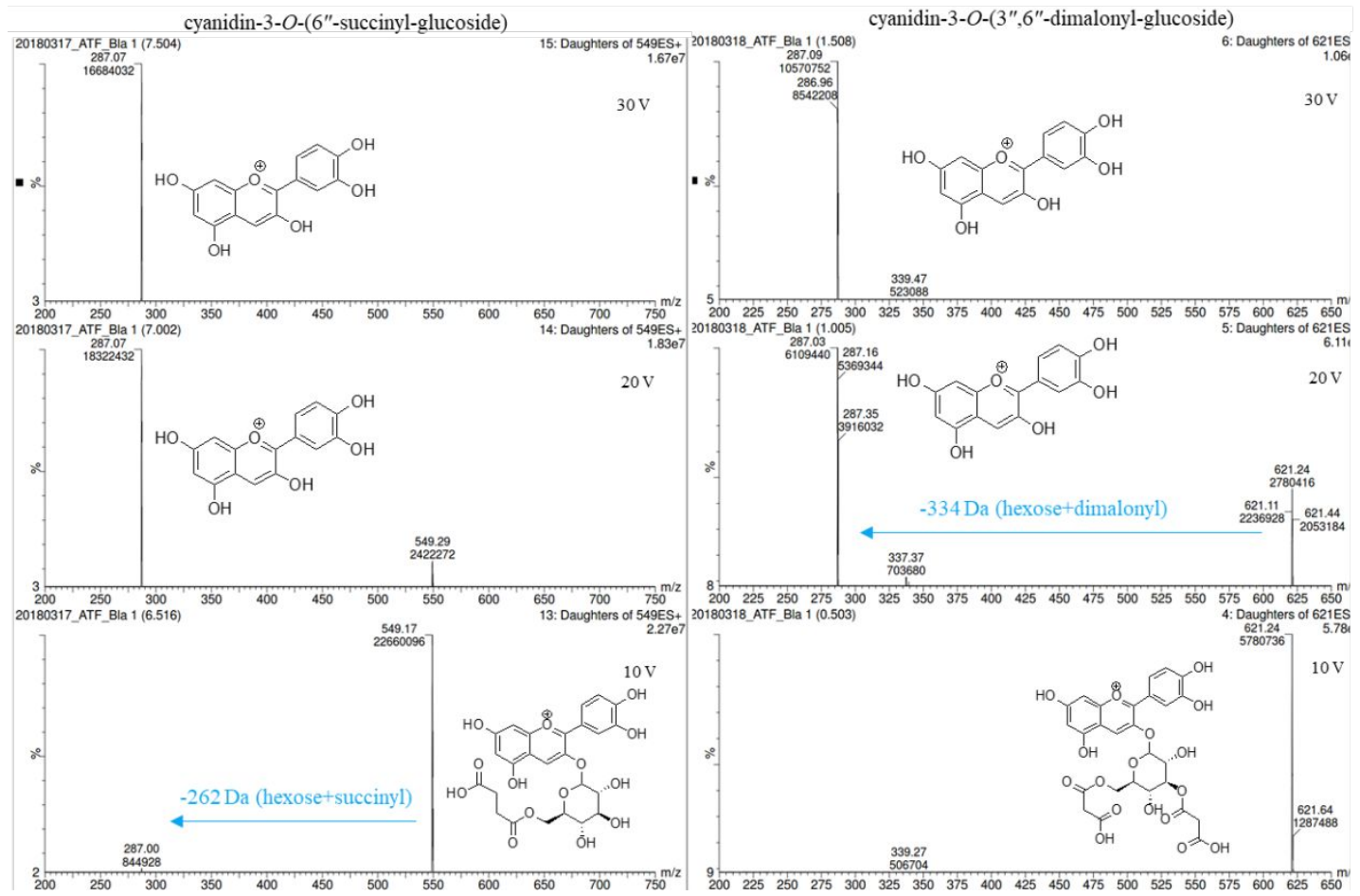

Figure S6. Mass spectra for cyanidin-3(6"-succinyl)- $O$-glucoside and cyanidin-3-O-(dimalonylglucoside). Data was obtained with Daughters method in MassLynx 4.1 using $10 \mathrm{~V}, 20 \mathrm{~V}$, and 30 V. Representative data is shown (sample ATF_Black).

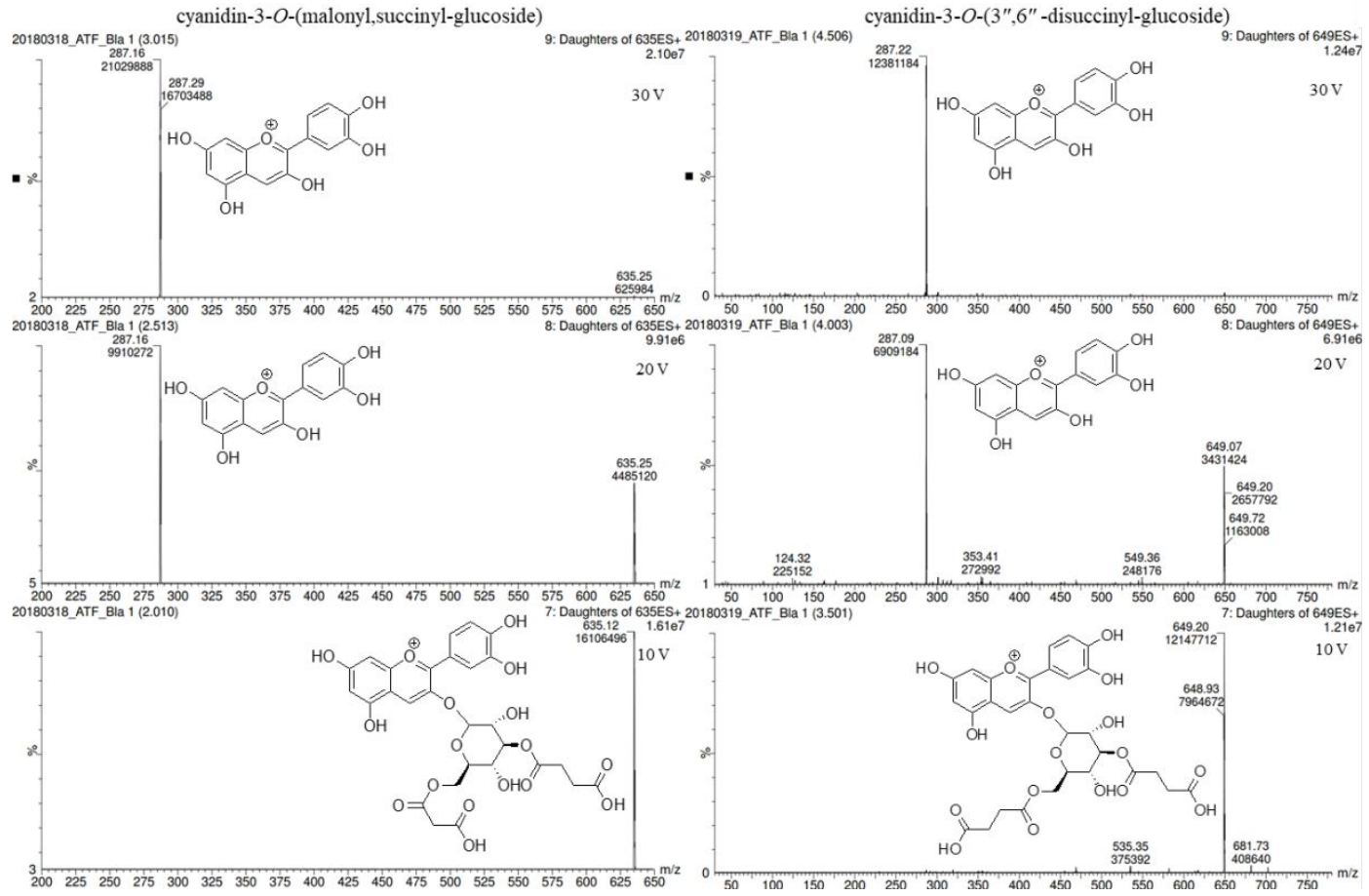

Figure S7. Mass spectra for cyanidin-3-O-(malonyl, succinyl-glucoside) and cyanidin-3-O(disuccinyl)-glucoside. Data was obtained with Daughters method in MassLynx 4.1 using 10 V, 20 $\mathrm{V}$, and $30 \mathrm{~V}$. Representative data is shown (sample ATF_Black). 


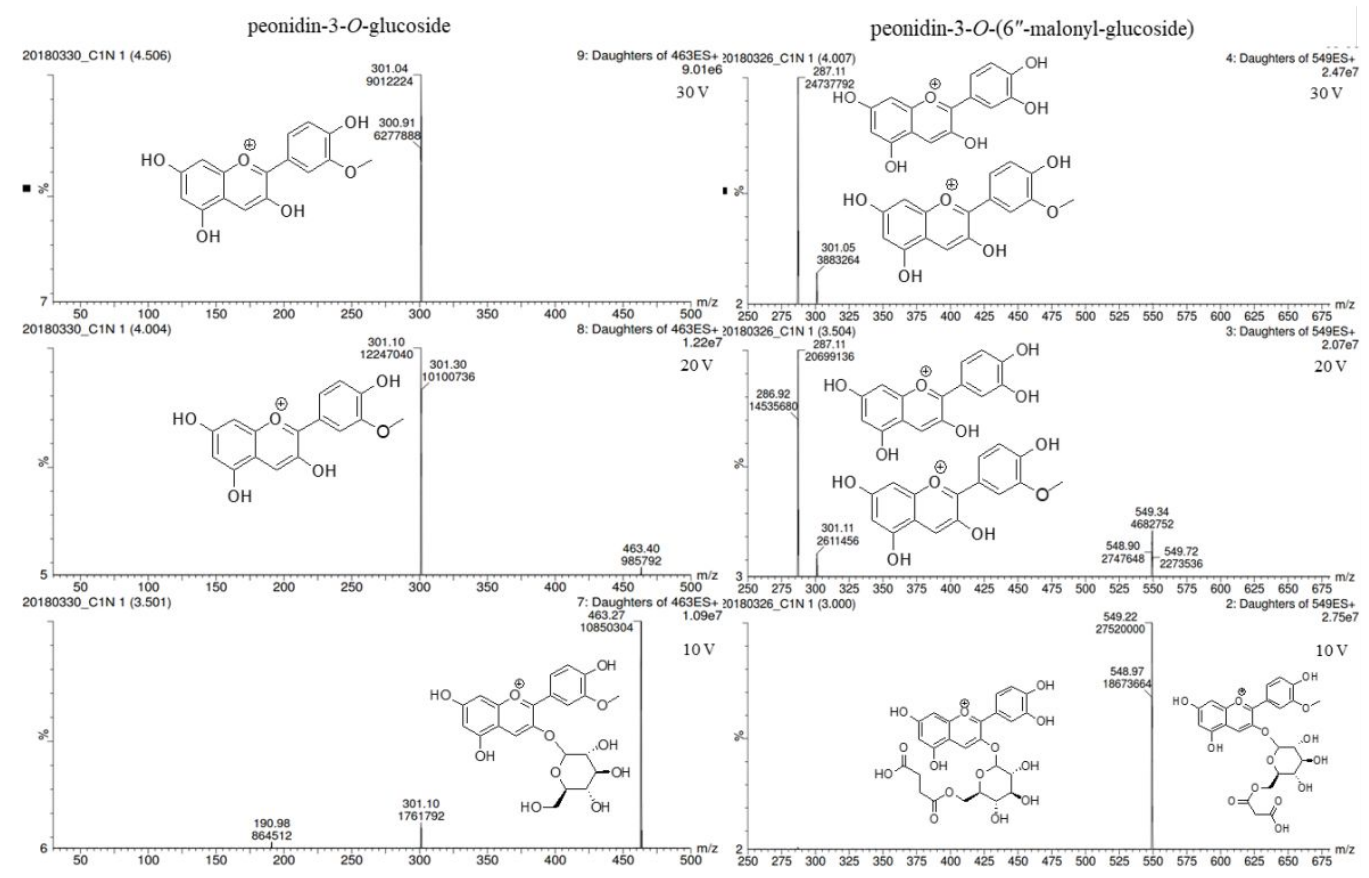

Figure S8. Mass spectra for peonidin-3-O-glucoside and peonidin-3-O-(6"-malonyl-glucoside) mixed with cyanidn-3-O-(6"-succinyl-glucoside). Data was obtained with Daughters method in MassLynx 4.1 using $10 \mathrm{~V}, 20 \mathrm{~V}$, and $30 \mathrm{~V}$. Representative data is shown (sample CN1_Black).

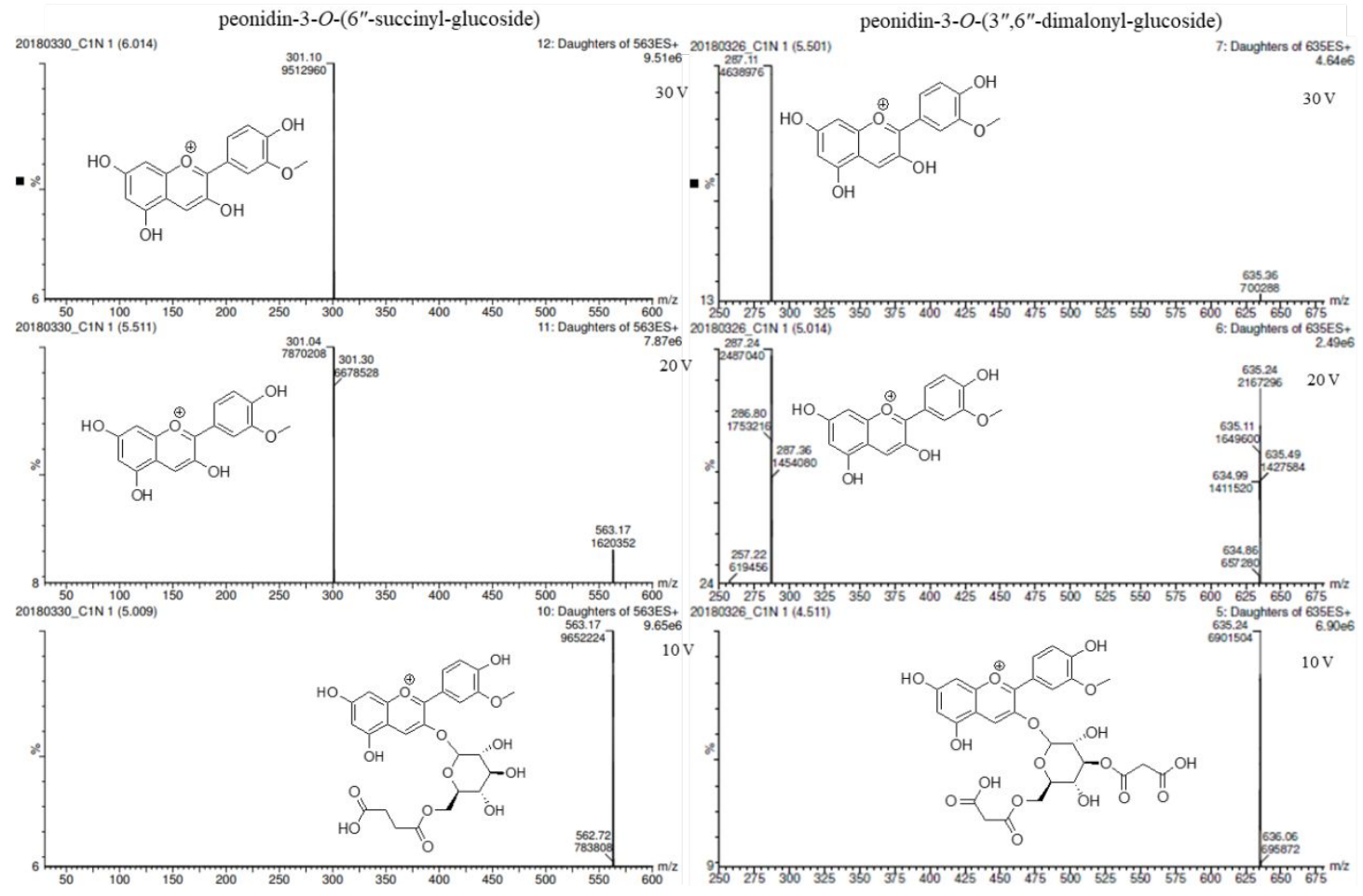

Figure S9. Mass spectra for peonidin-3-O-(6"-succinyl-glucoside) and peonidin-3-O-(malonyl, succinyl-glucoside) mixed with cyanidin-3-O-(disuccinyl-glucoside). Data was obtained with Daughters method in MassLynx 4.1 using $10 \mathrm{~V}, 20 \mathrm{~V}$, and $30 \mathrm{~V}$. Representative data is shown (sample CN1_Black). 


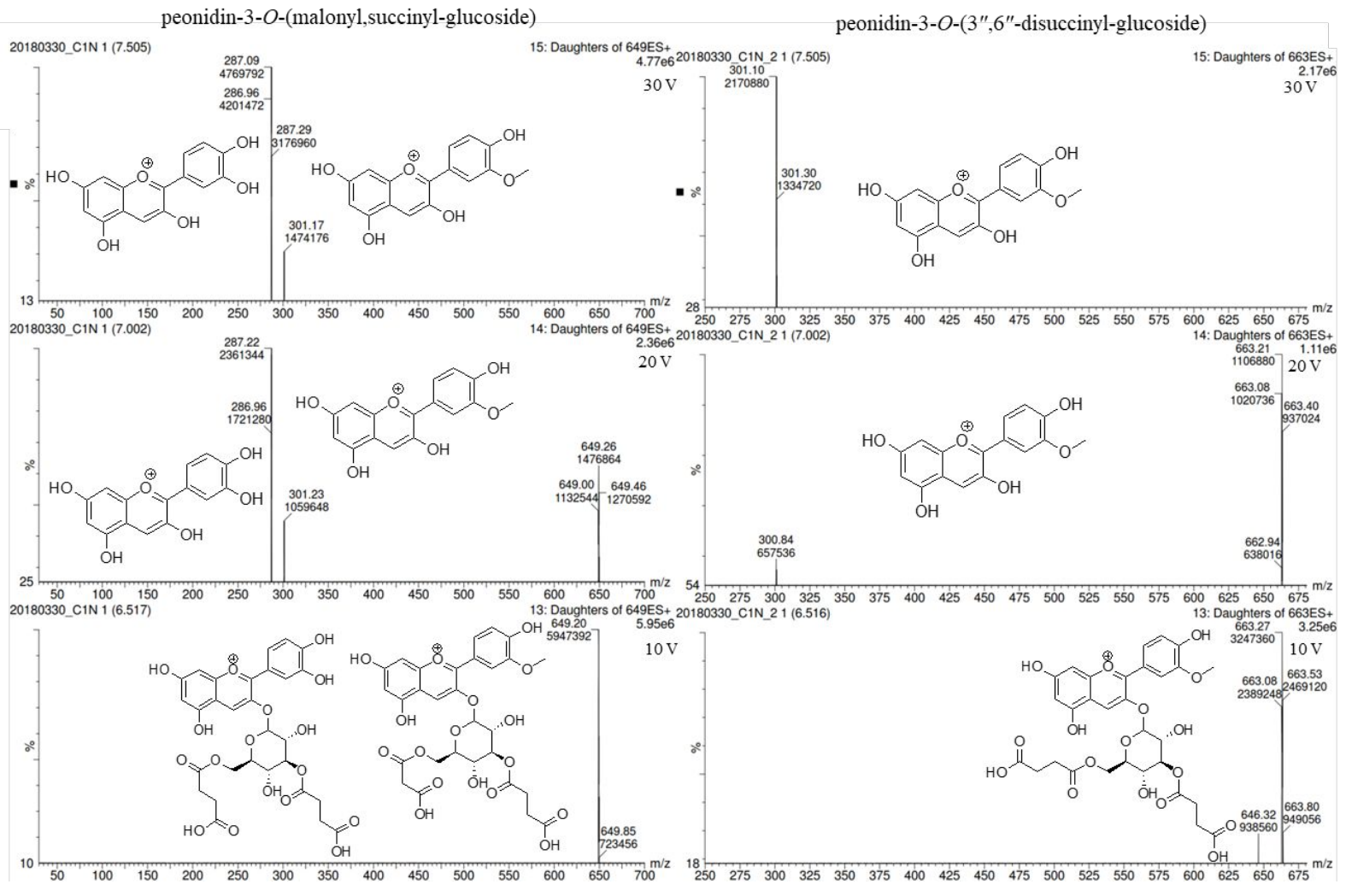

Figure S10. Mass spectra for the presumed peonidin-3-O-(malonyl-glucoside) and cyanidin-3-O(disuccinyl-glucoside) and peonidin-3-O-(disuccinyl-glucoside) at $649 \mathrm{~m} / z$ and $663 \mathrm{~m} / z$, respectively. The mass spectra were obtained with Daughters method in MassLynx 4.1 using $10 \mathrm{~V}$, $20 \mathrm{~V}$, and $30 \mathrm{~V}$. The sample used was CN1_Black 

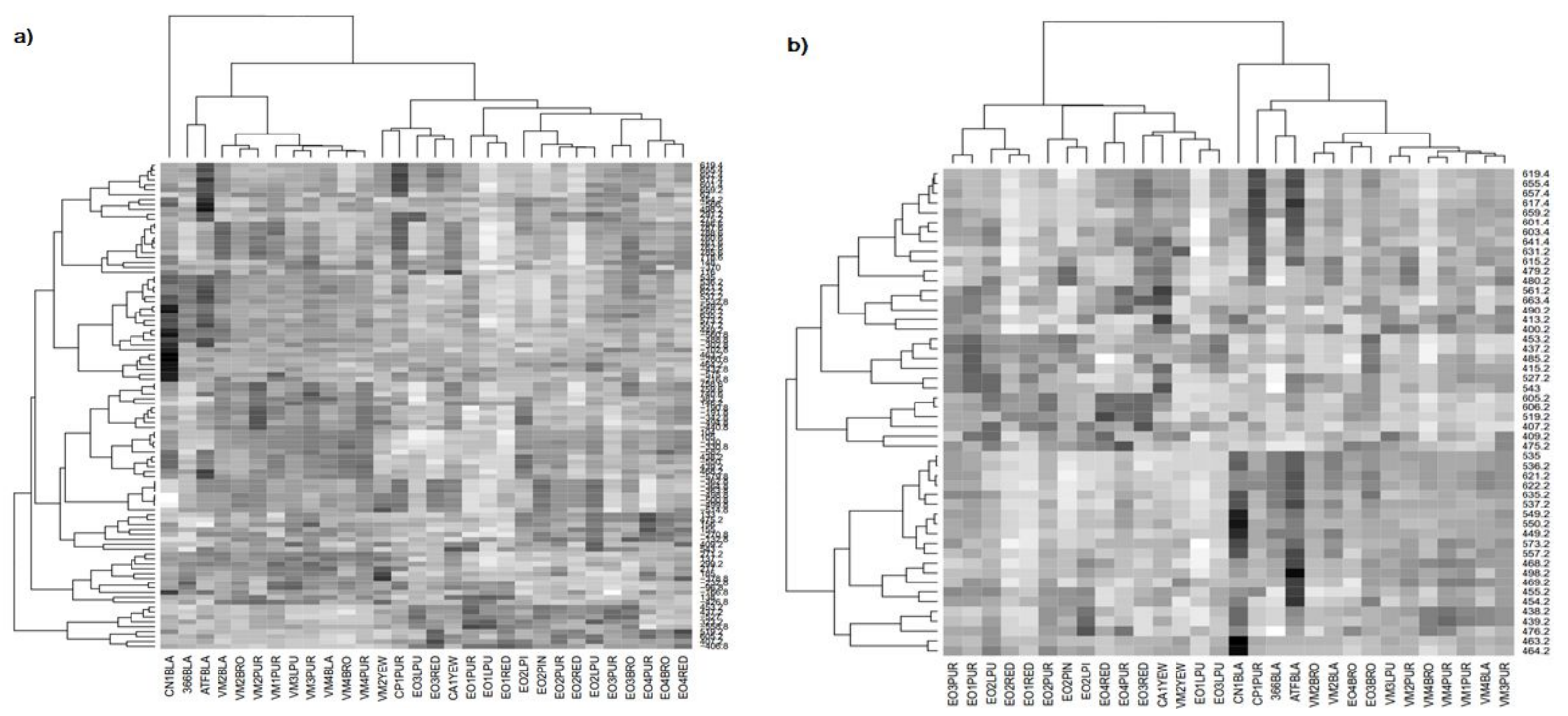

Figure S11. Heat maps using the most significant ions according to their $p$-value for the entry factor in the ANOVA model. In figure a) 100 ions detected in positive and negative ionization mode. It shows three clusters: one for Vitamaize and another one for "Elote occidental" landrace, while "Conico negro" is alone as a third clusted. In Figure b) the hierarchical clustering was done selecting ions only from the range $400-700 \mathrm{~m} / \mathrm{z}$. It forms two clusters: one of samples with high anthocyanin concentration which included Vitamaize and brown colored kernels from "Elote occidental", purple grain from "Pozolero purpura", and "Conico negro". The second cluster grouped the rest of the samples: pale, red and purple colored landraces. 


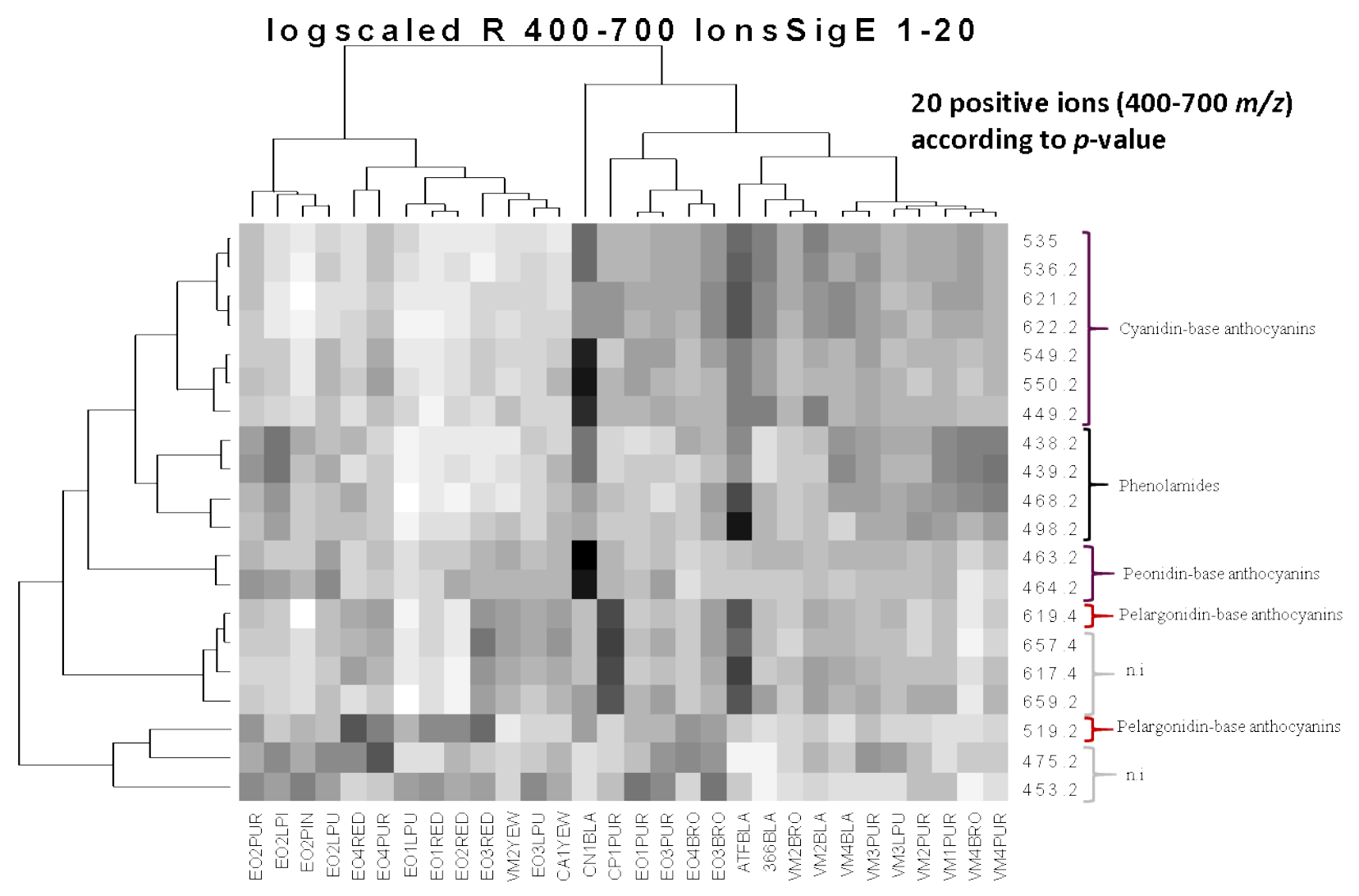

Figure S12. Heatmap using the most significant ions according to their p-value for the entry factor in the ANOVA model. The hierarchical clustering was done selecting the first 20 ions in the range from $400-700 \mathrm{~m} / \mathrm{z}$, which formed two clusters: one with higher anthocyanin concentration which included Vitamaize and brown colored kernels from "Elote occidental" purple grain from "Pozolero purpura", and "Conico negro". The second cluster grouped the rest: pale, red and purple colored genotypes. 


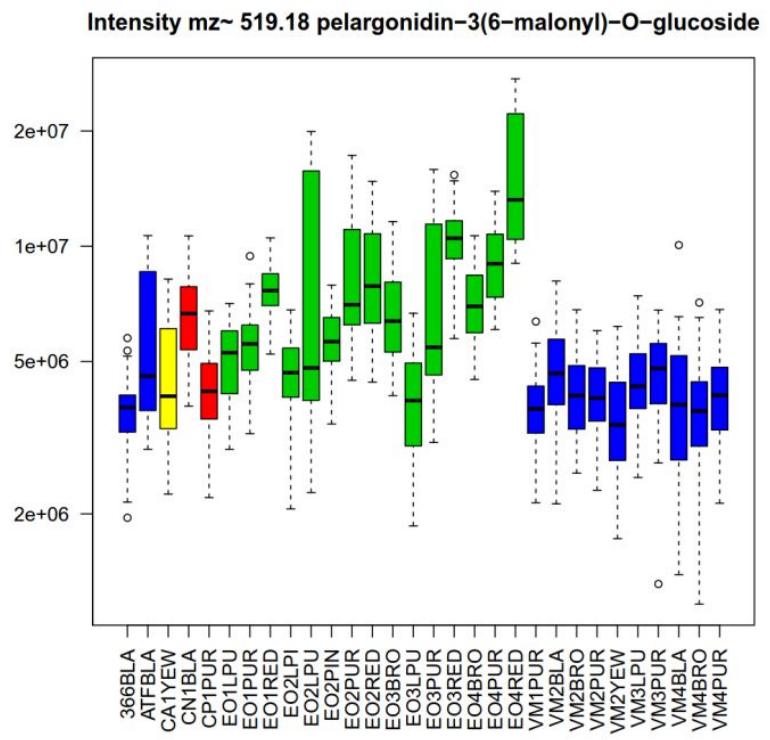

Figure S13. Boxplot distribution for pelargonidin-3-O-(6"-malonyl-glucoside) among different maize genotypes. It shows higher signal intensities for the "Elote Occidental" landrace in comparison to the other genotypes. The rectangles represent the first and third quartiles (boxes) and the median value (midline). The bars indicate the minimum and maximum values, whereas empty points represent data outliers.

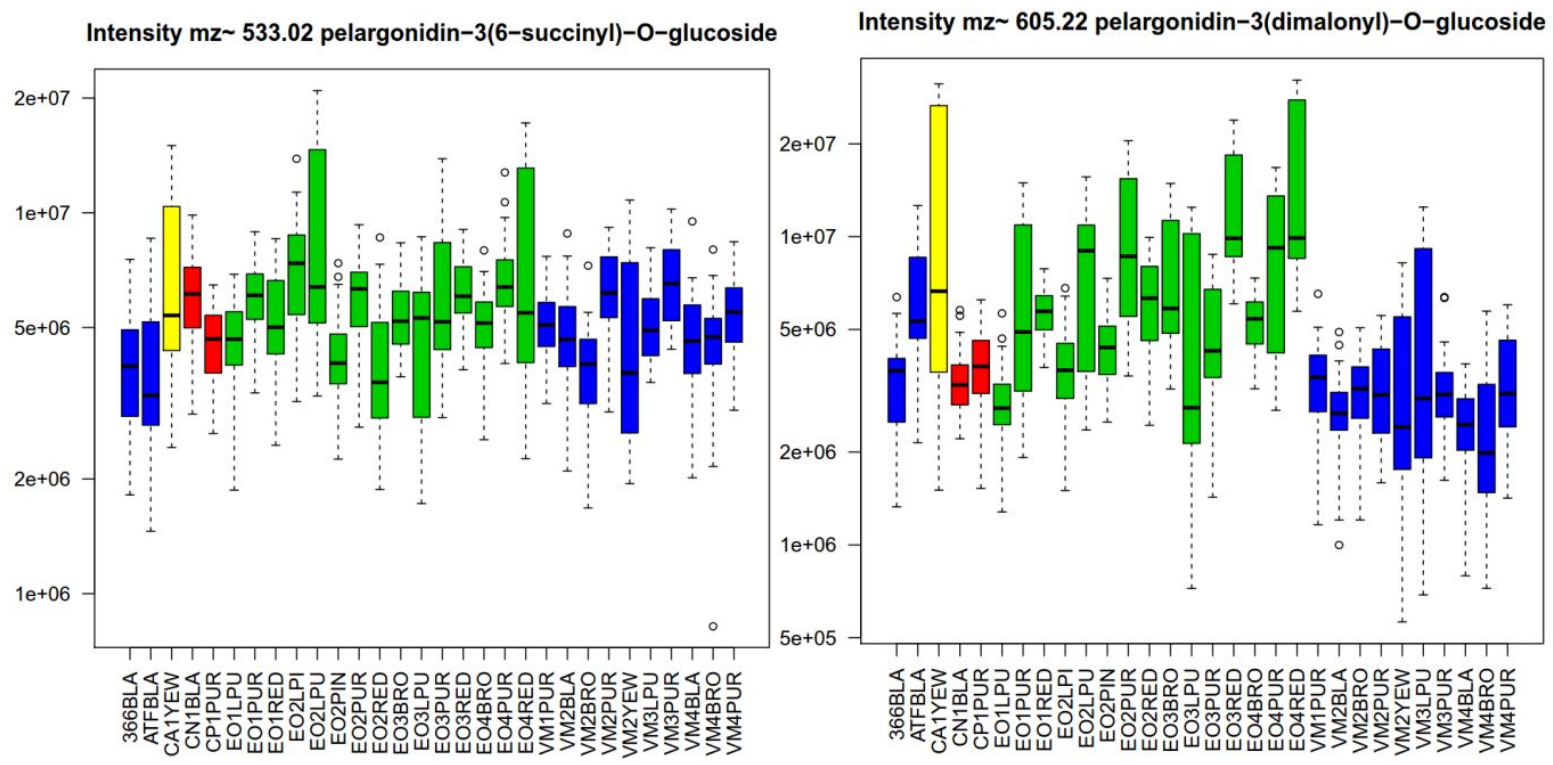

Figure S14. Boxplot distribution for ions corresponding to pelargonidin-3-O-(6"-succinyl-glucoside) and pelargonidin-3-O-(dimalonyl-glucoside). 

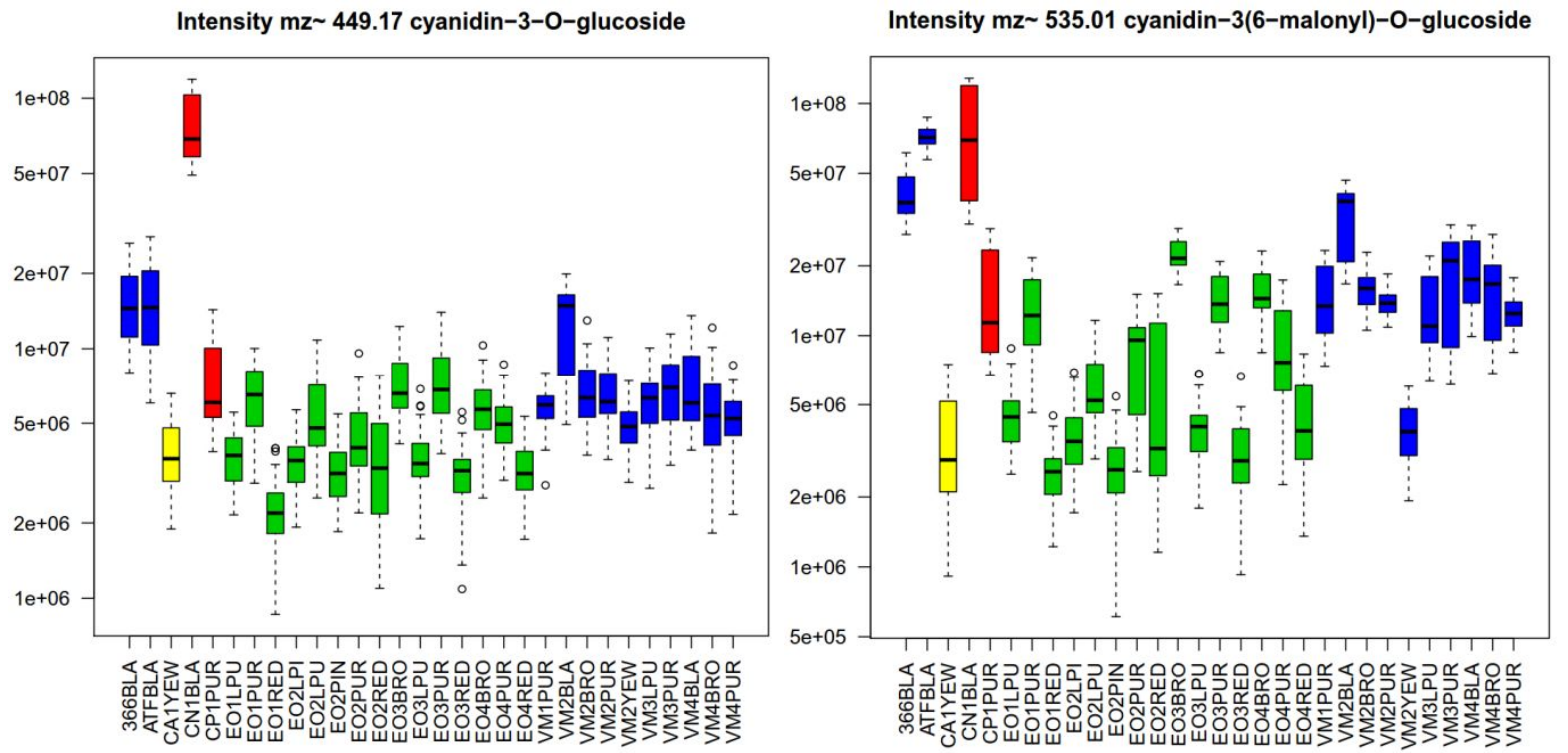

Figure S15. Boxplot distribution for ions corresponding to cyanidin-3-O-glucoside and cyanidin-3-O(6"-malonyl-glucoside). Higher intensities correspond to kernel colors black, purple, and brown but Ven greater in CN1_Bla and vitamaize lines 366_Bla and ATF_Bla. Red and pale color kernels presented the lowest intensities.

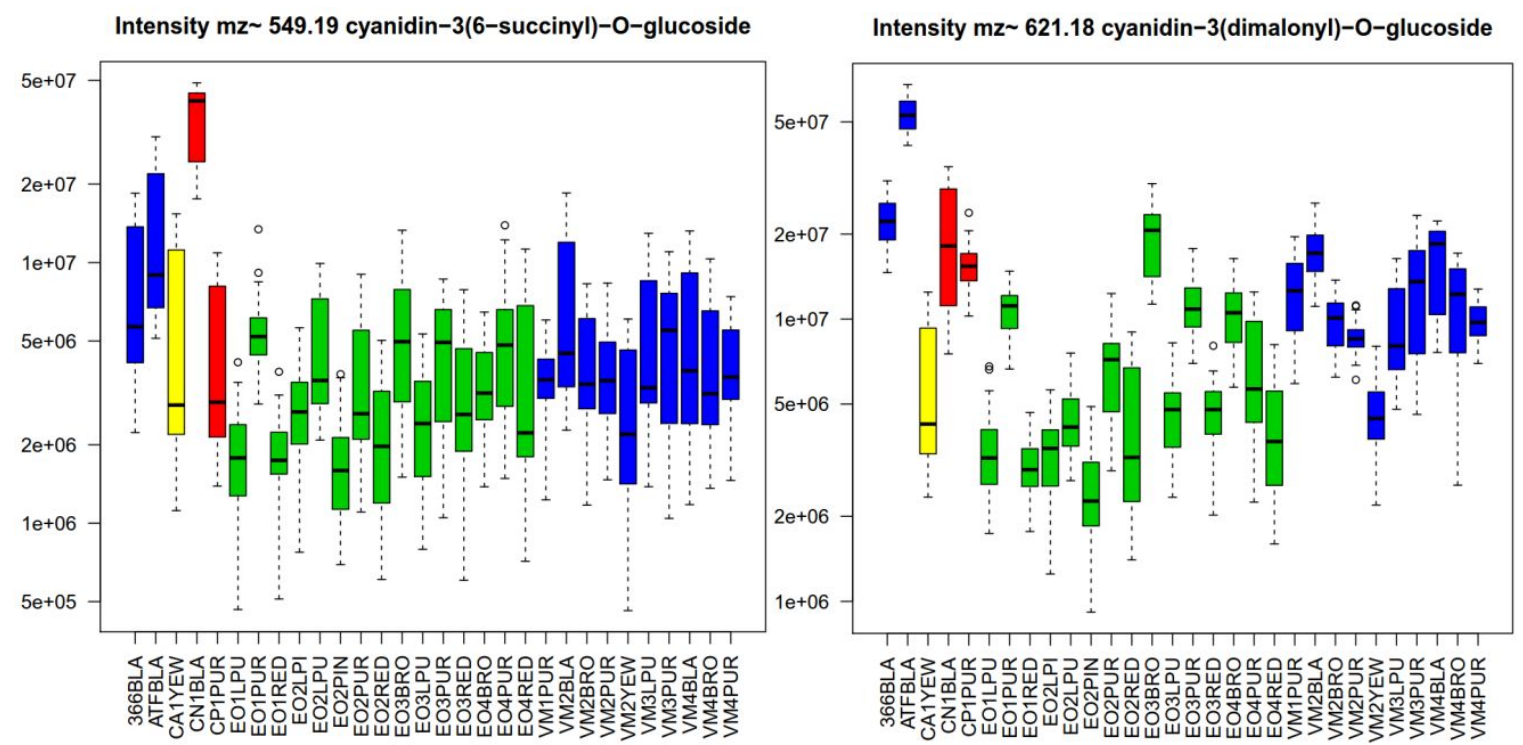

Figure S16. Boxplots distribution for cyanidin-3-O-(6"-succinyl-glucoside) and cyanidin-3-O(dimalonyl-glucoside). These boxplots shown that higher intensities in both ions correspond to kernel colors black, purple, and brown but Ven greater in CN1_Bla and vitamaize lines as 366_Bla and ATF_Bla. Red and pale color kernels presented lower intensities. 


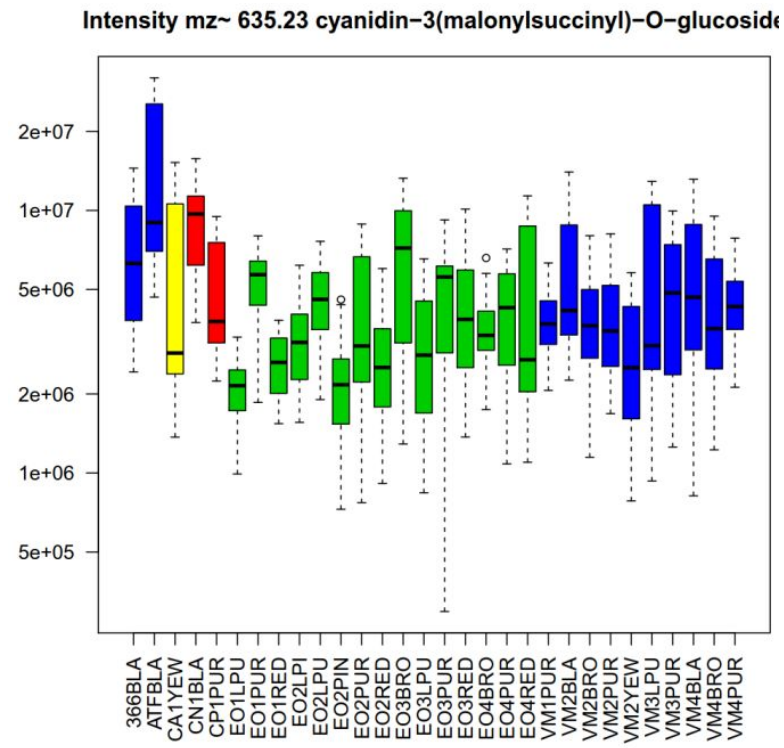

Figure S17. Boxplot distribution for cyanidin-3-O-(malonyl-succinyl)-glucoside. The ion had greater intensities in kernel colors like black, purple, and brown but Ven greater in CN1_Bla and vitamaize lines as 366_Bla and ATF_Bla. Red and pale color kernels presented the lower intensities.
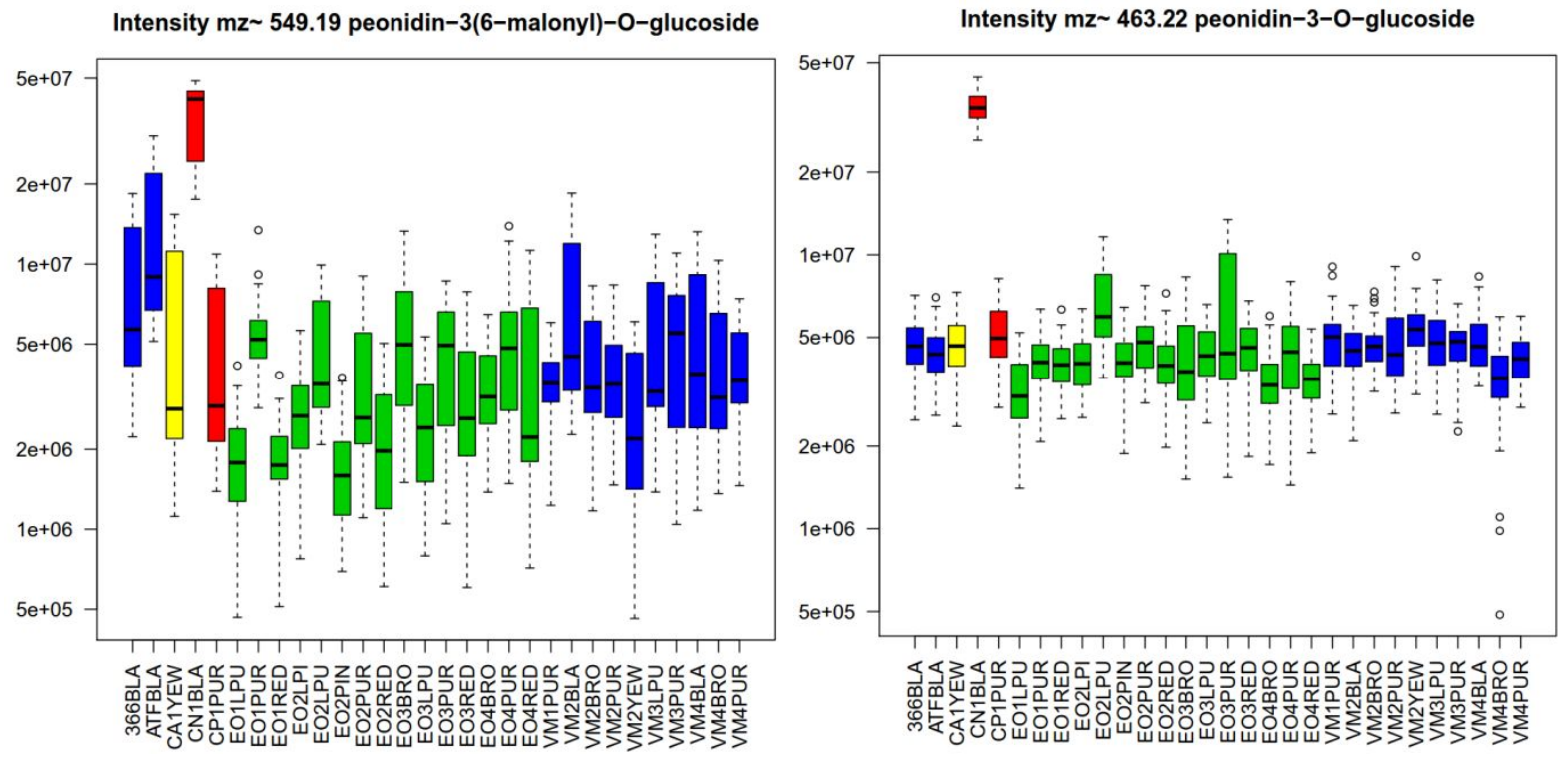

Figure S18. Boxplots for peonidin-3-O-(6"-malonyl-glucoside) and peonidin-3-O-glucoside. Both ions were highest in the CN1_Bla genotype. 